\title{
MODELAGEM E MAPEAMENTO TERMO-FLUIDODINÂMICOPARA O MONITORAMENTOE CONTROLEDA CORROSÃO EM ESCAPAMENTOS AUTOMÓTIVOS POR SENSORES ÓPTICOS EM REDE*
}

\section{Resumo}

Malu Braga Leal ${ }^{1}$ Ruben Miranda Carrilo ${ }^{2}$ Rogério Fernandes Brito ${ }^{3}$ José Carlos de Lacerda ${ }^{4}$ Ricardo Luiz Perez Teixeira ${ }^{5}$

Este trabalho propõe o controle e monitoramento da corrosão em escapamentos de automóveis pela modelagem e mapeamento termo-fluidodinâmico do escapamento utilizando o programa computacional de análise de volumes finitos ANSYS. A corrosão no escapamento do automóvel é produzida e catalisada pelos gases de combustão do motor que fluem aquecidos pelo escapamento. Ao se propor a utilização de uma rede de sensores ópticos nas regiões mapeadas pelo modelo obtido fluido dinâmico do escapamento do automóvel, tem-se uma ferramenta em tempo real para a visualização das regiões de maior gradiente térmico e mais suscetíveis à corrosão pelos gases de combustão no escapamento. A utilização do mapa termo fluidodinâmico do escapamento pelo programa ANSYS possibilita, assim, um maior controle das condições de corrosão no escapamento de um automóvel e na escolha de materiais mais adequados para a sua produção e funcionamento.

Palavras-chave: Monitoramento e controle de corrosão; Modelamento fluido dinâmico; Escapamento de automóvel.

\section{THERMAL FLUID DYNAMICMAPPING APPROACH TO OPTICAL SENSORS NETWORKED TO MONITORING AND CONTROL CORROSION IN CAR EXHAUSTS Abstract}

This paper attempts to propose a way of monitoring and controlling corrosion in car exhausts from a network of optical using a spatial thermal and fluid dynamic modeling approach by the analysis of finite volume software ANSYS. The use of the optical sensor network is due to the possibility of visualization of the corrosion occurring in the exhausts and the recognition areas most affected by the oxidation process in the exhaust, because it is a sensor that can work in various hostile environments, from high pressure, high temperature humid environments without creating damage to its structure and jeopardize the results, which will facilitate for information gathering around the exhaust, then seek recognition of the most affected areas for the placement of sensors, this system soon will help to do a control of this process.

Keywords: Monitoring and controlling corrosion; Fluid dynamic modeling; Automobile exhaust.

1 Graduando em Engenharia Mecânica, $7^{\circ}$ período, UNIFEI - Universidade Federal de Itajubá Campus Itabira, Itabira, MG, Brasil.

2 Engenheiro Mecânico, M.Sc. Engenharia Mecânica pela UNIFEI, professor, Universidade Federal de Itajubá- Itabira, Curso de Engenharia Mecânica, Itabira, Brasil.

3 Engenheiro Mecânico, Dr. Engenharia Mecânica pela UNIFEI, professor, Universidade Federal de Itajubá- Itabira, Curso de Engenharia Mecânica, Itabira, Brasil.

4 Engenheiro Industrial Mecânico, M.Sc. Engenharia de Materiais pela UFOP, professor, Universidade Federal de Itajubá- Itabira, Curso de Engenharia Mecânica, Itabira, Brasil.

5 Engenheiro Químico, Dr. em Ciências, Engenharia Metalúrgica e de Materiais pela UFRJ, professor de materiais metálicos, Universidade Federal de Itajubá- Itabira, Curso de Engenharia de Materiais, Itabira, Brasil. 


\section{INTRODUÇÃO}

O escapamento é uma peça obrigatória nos automóveis, sua ausência e inequidade pode ser avaliada pelas autoridades competentes, e na forma estabelecida pelo Conselho Nacional de Trânsito (CONTRAN), como infração grave sujeita a penalidade de multa e medida administrativa de retenção do veículo para regularização; conforme o artigo 230 incisos IX a inciso XI. O escapamento também deve cumprir com a obrigatoriedade do controle de emissão de gases e ruídos pelo automóvel, segundo a lei federal brasileira $n^{\circ}$ 9.503, de 23 de setembro de 1997 para a legislação do código de trânsito brasileiro (CTB) artigos 104 e 105 [1].

O escapamento de automóvel, além da obrigatoriedade e regularidade de funcionamento pela legislação brasileira, tem relevância econômica nacional na participação de montadoras e fabricantes de autopeças, pois sem escapamento não se pode vender automóveis.Em termos de Produto Interno Bruto (PIB) brasileiro, a participação de veículos automotores foi próxima de $19 \%$ em 2013 , segundo dados da Associação Nacional dos Fabricantes de Veículos Automotores (ANFAVEA) [2].

O escapamento, por se tratar de uma peça complexa por onde escoam gases de combustão aquecidos, possui áreas sujeitas as mais diversas influências corrosivas como: ambiente externo, altas temperaturas, resíduos de saída e muitas outras interferências que podem levar às ações químicas erosivas do material metálico, ou seja, a corrosão e a danos ou a perda funcional ou estrutural da peça [3].

O modelamento e a simulação computacional termo fluidodinâmica dos gases de combustão no escapamento permite um melhor estudo e controle da ação corrosiva no escapamento, estendendo o tempo de vida de seu uso no automóvel. O controle da corrosão por sensores óticos em rede nos pontos de maior gradiente térmico visa a monitorando as regiões de maior efeito térmico catalítico de corrosão (mais sujeitas à corrosão ou de danos a peça) em contato com os gases de combustão. Os sensores óticos em rede podem levar feixes luminosos por grandes extensões, sem perda de informação o que irá facilitar para o recolhimento de informação em todo o escapamento, facilitando assim no reconhecimento de todas as áreas. Logo esse sistema nos permitirá encontrar os pontos de maior corrosão e a fazer um controle deste processo e numa melhora do tempo de vida útil do escapamento [4].

\section{MATERIAIS E MÉTODOS}

No modelamento para o escoamento de gases de combustão em escapamento de automóvel foi utilizado o modelo do escapamento do veículo Volkswagen modelo Gol GTI geração 4 (VW Gol GTI) disponível na Universidade Federal de Itajubá Campus Itabira. O modelo VW Gol GTI foi escolhido pela sua grande relevância no quadro produtivo automobilístico brasileiro [5]. Após a escolha do tipo e do modelo de escapamento, fez-se as medições das dimensões espaciais diretamente no escapamento do VW Gol GTI, sendo utilizado para a sua realização uma trena e um paquímetro de precisão. A partir das medidas obtidas diretamente do escapamento do VW Gol GTI, fez-se o desenho do escapamento em CAD utilizando o programa SolidWorks ${ }^{\circledR}$.

O desenho do escapamento no CAD foi formatado na extensão do ParaSolid para o uso no pacote de modelamento computacional CFD do programa ANSYS.

Para a análise do problema no CFD, realizou-se a discretização termo fluidodinâmica da condução de gás de condução, da convecção de gás de 
combustão e da condução para a carcaça foi estabelecido modelo computacional e as funções que geram o problema.

Em seguida, fez-se a escolha e o desenvolvimento da malha mais adequada para o escapamento, onde fosse possível um maior detalhamento das condições e da geração de resultados confiáveis e que convergissem. Estabeleceu-se antes da simulação computacional, então, as condições de contorno do problema em estudo, dados de contorno obtidos a partir dos ensaios experimentais diretos do VW Gol GTI. As condições de contorno principais de velocidade, temperatura e pressão dos gases de exaustão (combustão) obtidas pelas referências e medições na saída do escapamento em estudo, permitiu o cálculo da vazão de gás de combustão.

As condições iniciais de temperatura e vazão forma realizadas utilizando termômetro e anemômetro digital de acordo com algumas rotações típicas de funcionamento de automóvel. As medições pelo termômetro e anemômetro foram tomadas diretamente no escapamento com o veículo parado nas diversas rotações citadas. O veículo parado, apresenta a pior condição possível para a transferência de calor, pois não há trocas (perdas) significativas de calor por convecção para o ar ambiente, decorrendo assim condições de maior gradiente térmico nas diversas partes do sistema de escapamento. O instrumento empregado na medição da temperatura foi o termômetro infravermelho digital portátil, da INSTRUTHERM, modelo TI-920, com uma resolução de $0,1^{\circ} \mathrm{C}$, escala de $-50^{\circ} \mathrm{C}$ a $1600^{\circ} \mathrm{C}$ e uma precisão de $\pm 1,5 \%$ da escala leitura da leitura, sendo o valor de cerca de $\pm 2{ }^{\circ} \mathrm{C}$. A emissividade térmica usada no termômetro foi a indicada de 0,95 , por se tratar da emissividade térmica dos aços foscos, material utilizado no escapamento do VW Gol GTI. O instrumento empregado na medição da velocidade de vazão dos gases de exaustão foi o termo anemômetro digital portátil, da INSTRUTHERM, modelo TAD-800, que possui uma resolução de 0,01 numa escala de $0,60 \mathrm{~m} / \mathrm{s}$ a $30,00 \mathrm{~m} / \mathrm{s}$ e erro de $\pm 3 \%$ da escala, sendo o valor de cerca de $\pm 0,20 \mathrm{~m} / \mathrm{s}$.

Com a escolha do tipo e modelo de escapamento e as condições de fronteira retiradas diretamente do veículo em funcionamento, fez-se a simulação computacional para avaliar a convergência termo fluidodinâmica do modelo ANSYS CFX empregado com as condições reais de contorno do veículo estudado. $O$ resultado obtido é um mapa termo fluidodinâmico do escapamento indicando as regiões de maior gradiente térmico durante o funcionamento do veículo e onde se colocará os sensores óticos em rede para o controle mais efetivo da corrosão dos gases de combustão catalisada pela temperatura.

\section{RESULTADOS E DISCUSSÃO}

\subsection{Desenho Solidworks}

$\mathrm{Na}$ Figura 1, tem-se o desenho do escapamento do Gol GTI realizado com o uso do programa SolidWorks ${ }^{\circledR}$ na visão isométrica. O desenho foi exportado para o programa ANSYS CFX no formato Parasolid utilizando as mesmas cotas ou dimensões conforme escapamento original. 


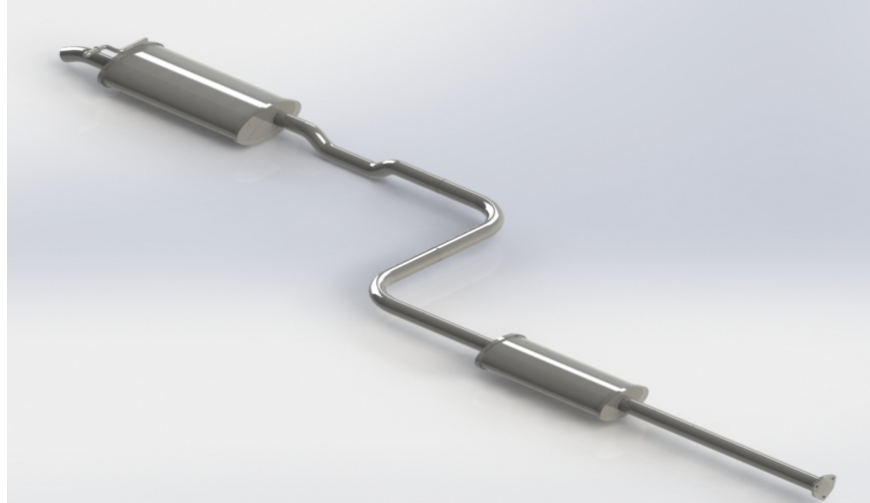

Figura 1 - Desenho do escapamento realizado no programa SolidWorks ${ }^{\circledR}$.

\subsection{Modelamento Termodinâmico Gráficos}

Nas Figuras 2 e 3, tem-se o escapamento do VW Gol GTI realizado com o uso do programa SolidWorks ${ }^{\circledR}$ com a malha no interior do escapamento sendo feita a partir do ANSYS. A malha foi desenvolvida nas regiões internas do escapamento, sendo o escapamento encapsulado e desconsiderado o material do mesmo, isto foi realizado para que a malha e a simulação fossem feitas apenas nas regiões de estudo do escapamento, nas regiões de maior relevância.

O desenho em malha foi desenvolvido segundo condições reais de fronteira de temperatura, pressão e vazão para os componentes de combustão gasosos oriundos do motor, isto foi necessário para a validação da simulação fluido termodinâmica, pois é necessária a convergência de resultados de simulação com os dados de fronteira reais para a validação do modelo e do mapa obtido para o escapamento. Os resultados da simulação produziram um modelo termodinâmico de gradiente de temperatura, pressão e velocidade de escoamento no tubo do escapamento, Figuras 4,5 e 6 . A malha utilizada no modelamento foi a malha tetraédrica, o tamanho dos elementos é de $2 \mathrm{~mm}$, como mostrado na seção do escapamento na Figura 3.

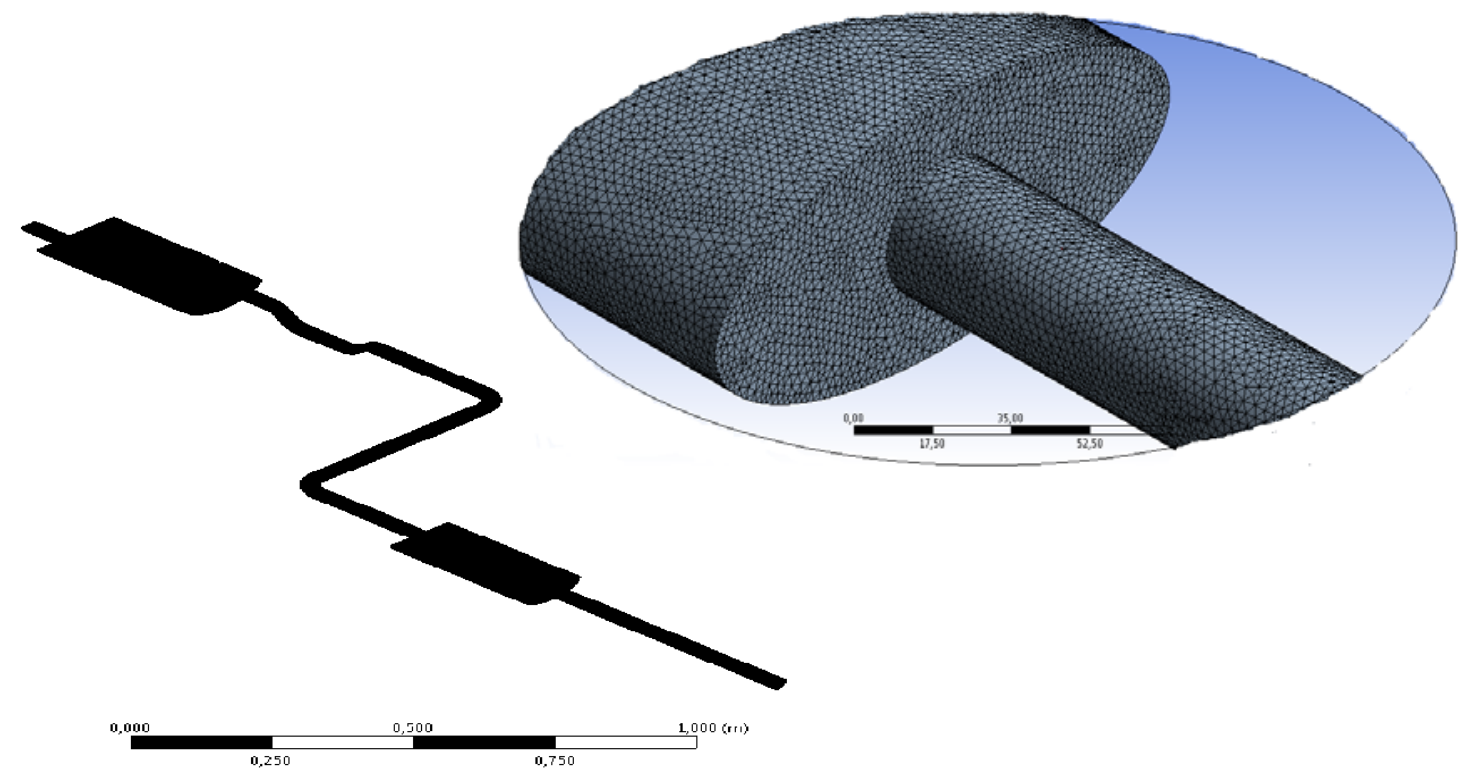

Figura 2 - Escapamento com a malha em detalhe. 


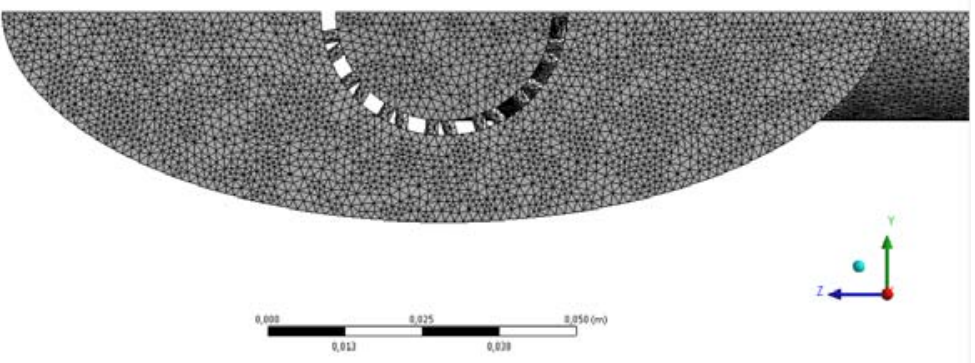

Figura 3 - Escapamento em corte com a malha.

\subsection{Gás Presente no Interior do Escapamento}

Conceitualmente, a gasolina comum, tipo "C", é o combustível, usualmente, utilizado para automóveis (e neste trabalho) e seu teores máximos de etanol e enxofre são estabelecidos pela ANP (Agência nacional do Petróleo, Gás Natural e Biocombustíveis), ANP $n^{\circ} 40$, de 25.10.2013, onde é estabelecido as especificações para a comercialização de gasolina automotivas e sua qualidade, especificando teor máximo de $50 \mathrm{mg} / \mathrm{kg}$ de enxofre [8]. No entanto, o teor máximo de etanol anidro combustível, foi estipulado pela legislação vigente, desde $1^{\circ}$ de maio de 2013 sendo de 25\%, conforme Portaria MAPA (Ministério da Agricultura, Pecuária e Abastecimento) $n^{\circ} 105$ e Resolução CIMA (Conselho Interministerial do Açúcar e do Álcool) n_ 1, de 28 de fevereiro de 2013 [6]. Considerou-se a combustão deste gás da combustão de gasolina $C$ no motor, pois o escapamento estudado não apresenta catalisador, segue a equação da combustão.

Logo, temos:

\section{$0,749999 \mathrm{C}_{\mathrm{v}} \mathrm{H}_{13}+0,25 \mathrm{C}_{2} \mathrm{H}_{\mathrm{b}} \mathrm{OH}+0,0000007805 \mathrm{SO}_{2}+13,39127055\left(\mathrm{O}_{\mathrm{i}}+3,76 \mathrm{~N}_{\mathrm{i}}\right)$ $+28,02474529 \mathrm{H}_{2} \mathrm{O} \rightleftarrows 3,249997 \mathrm{CO}+0,0000007805 \mathrm{SO}_{2}+3,249997 \mathrm{CO}_{2}+$ $35,52467429 \mathrm{H}_{2} \mathrm{O}+50,35117727 \mathrm{~N}_{2}+4,89131105 \mathrm{O}_{2}$

Pela equação anterior [1], obteve-se, então a porcentagens moleculares de cada elemento e ao gás após a combustão. Ao obter-se as porcentagens oriundas da combustão, material presente no escapamento, realizou-se a simulação das passagens dos gases pelo escapamento, o que permitiu a identificação dos pontos de maiores temperaturas e a visualização do gradiente de temperatura do mesmo, Figuras 4,5 e 6 . 


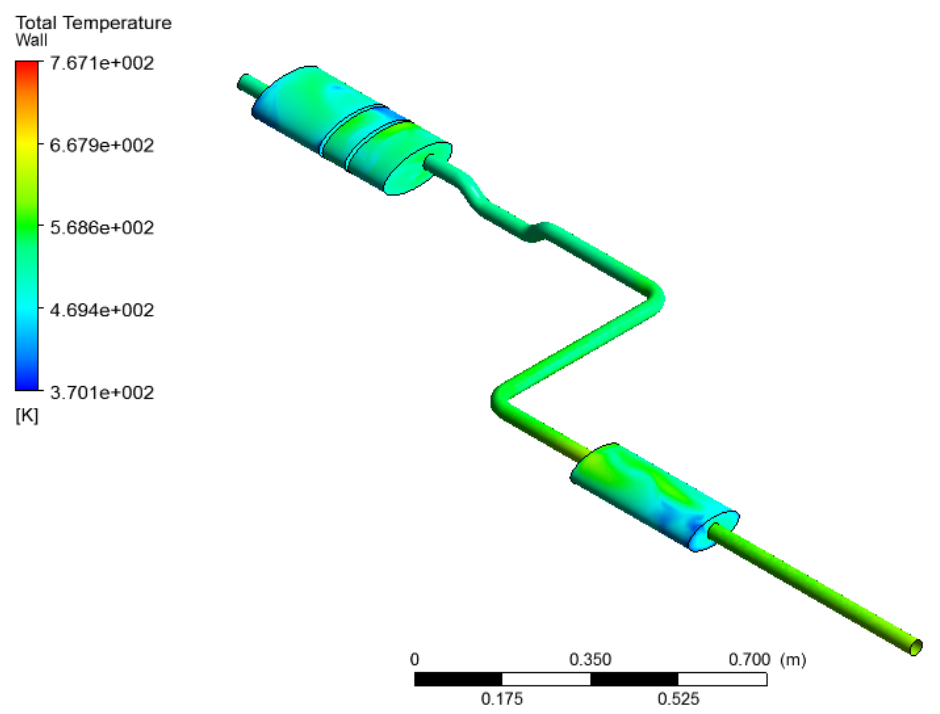

Figura 4 - Gradiente de temperatura no escapamento.

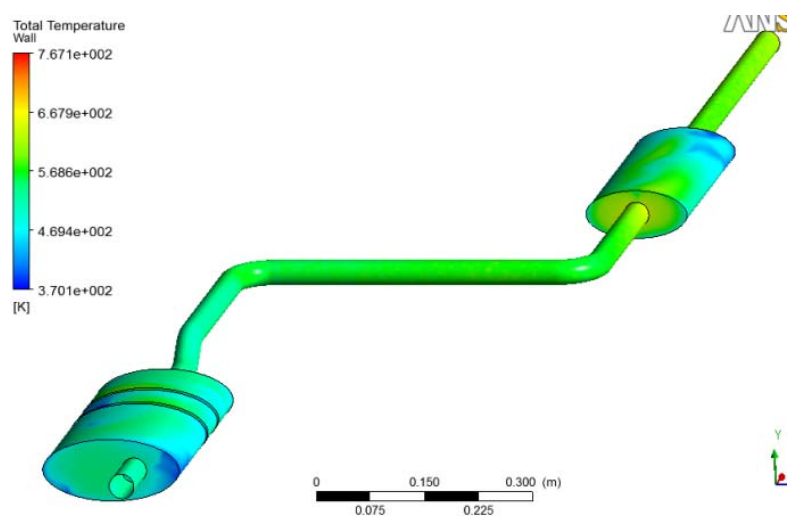

Figura 5 - Gradiente de temperatura.

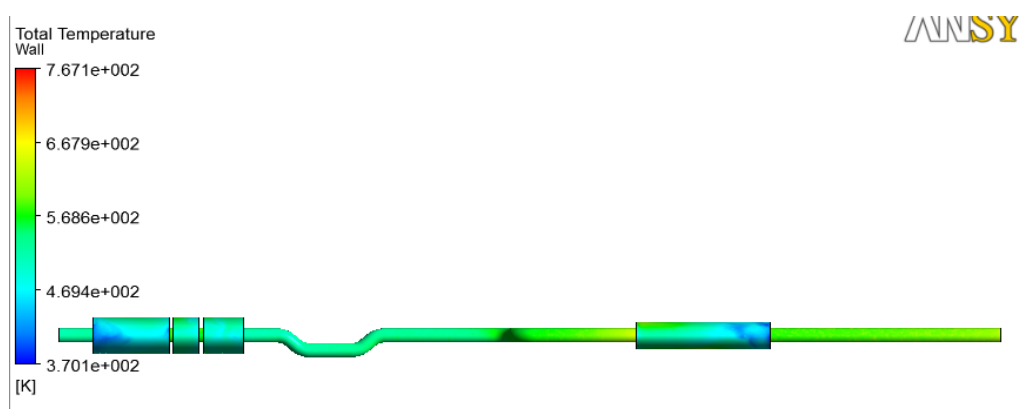

Figura 6 - Gradiente de temperatura no escapamento.

Após a identificação das regiões de maior gradiente térmico pelas Figuras 4,5 e 6 , fez-se o virtual posicionamento dos sensores óticos de acordo com o projeto proposto, vislumbrando a identificação dos principais pontos de corrosão devido a uns dos principais fatores que maximizam e viabilizam o processo, que são as altas temperaturas, Figura 7. 


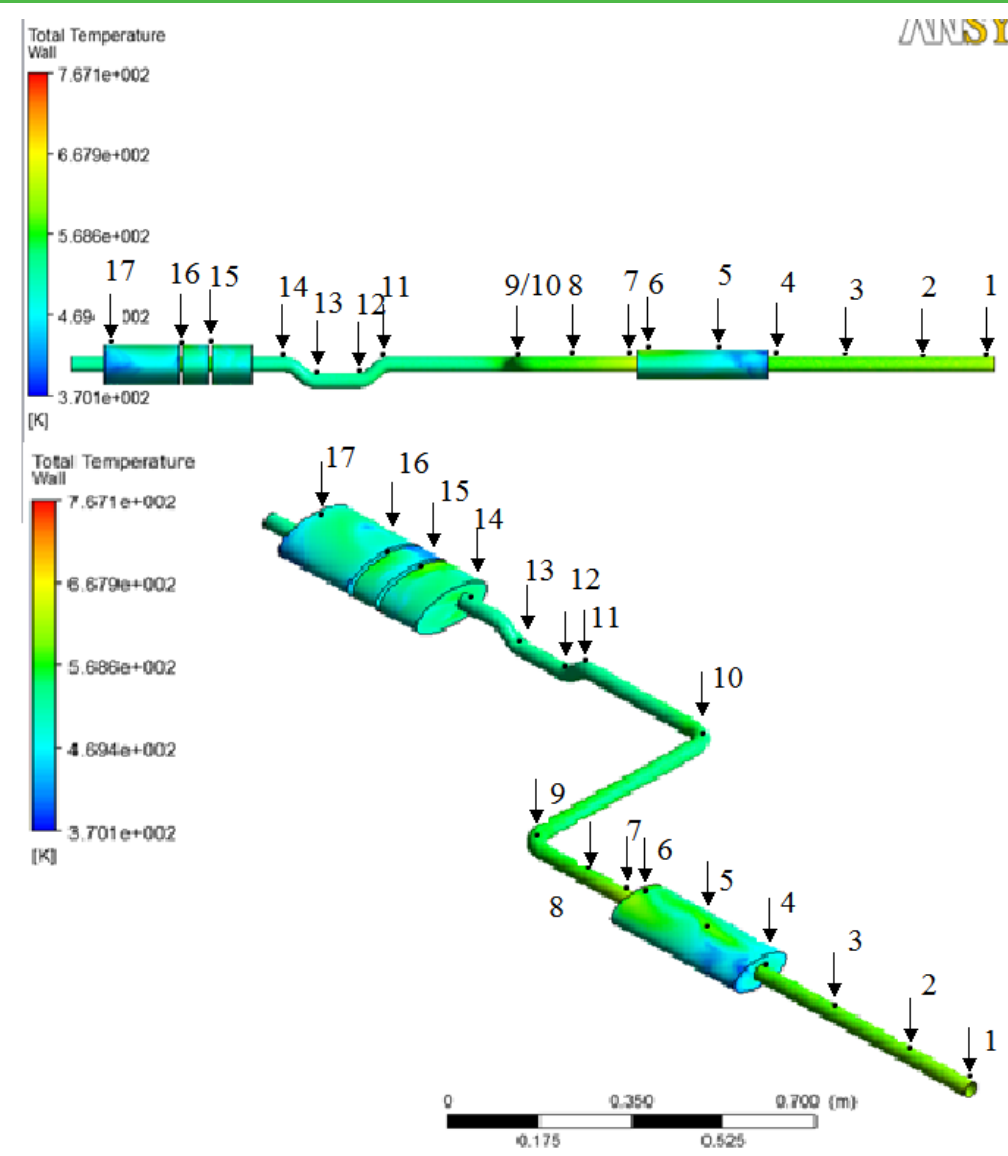

Figura 7 - Pontos de colocação dos sensores no escapamento.

Os pontos críticos para a colocação dos sensores óticos, Figura 7, foram dispostos da seguinte forma, após a avaliação do gradiente de temperatura, desenvolvendo este posicionamento devido as seguintes identificações:

- $\quad$ Primeiramente, os pontos de sensores 1, 2 e 3 no tubo de entrada foram dispostos desta maneira, pois o fluido após ao fim da combustão entra no tubo de escapamento com alta temperatura, logo o monitoramento deve ser mais efetivo nesta parte.

- $\quad$ Nas entradas do abafador e silenciador, sendo os pontos onde ocorre alta temperatura devido a colisão do fluido nas paredes, pontos 4 e 14 .

- $\quad$ Nas saídas do abafador e silenciador, pontos 6, 7 e 17, pois ocorre um aumento de temperatura devido a diminuição de área, o que leva a um aumento de velocidade do fluido, com isso a colisão das partículas do fluido com as paredes.

- Nos pontos 5 e 8, foi colocado pontos de sensores, para o controle dentro da tubulação para controle continuo e para controle dos pontos internos.

- $\quad$ Nas curvaturas foram também colocados devido a mudança de direção dos fluidos e o encontro do fluido em alta velocidade com a parede da tubulação, como por exemplos os pontos 9, 10, 11,12 e 13 .

A utilização dos sensores óticos de corrosão nos pontos anteriormente amostrados em mapa pela Figura 7 possibilita à indústria automobilística ferramental adequado para a melhoria das condições e materiais empregados no funcionamento do automóvel VW Gol GTI (e que pode ser generalizado, de acordo com cada tipo de escapamento de automóvel) para a produção de veículos de melhor conformidade frente a legislação brasileira e de maior qualidade de materiais para a exaustão dos gases de combustão de combustível oriundos do motor pelo escapamento, ou de menor custo de reposição com o uso. 


\section{CONCLUSÃO}

O modelo e mapa termo fluidodinâmico computacional para o escapamento do automóvel VW Gol GTI foi obtido e está em condições para a disposição de sensores óticos para controlar e monitorar a corrosão diretamente em escapamentos de carros. O modelo tem um embasamento termo fluidodinâmico sendo o mapeamento obtido pelo programa de análise de volumes finitos ANSYS. A utilização do modelo apresentado fornece informações para o reconhecimento das áreas mais afetadas pela corrosão durante o funcionamento automotivo no escapamento sendo um referencial para a disposição espacial de sensores óticos para monitoração, controle e inibição do processo corrosivo em rede. O impacto na indústria automobilística possibilitará a produção de escapamentos, obrigatórios pela legislação brasileira no veículo automotor, com melhor qualidade e maior tempo de vida, ou de menor custo de reposição.

\section{Agradecimentos}

Agradecemos à Universidade Federal de Itajubá, ao grupo GPESE da Unifei-Itabira pelos professores líderes José Carlos de Lacerda e Valdir Tesche Signoretti e à Fundação de Amparo à Pesquisa do Estado de Minas Gerais (FAPEMIG) pelo apoio a esta pesquisa, PIBIC 2014/2015. Somos gratos também à Universidade Federal de Pernambuco pelos professores Joaquim Martins Filho e Jehan F. Nascimento e da Universidade Federal de Itajubá.

\section{REFERÊNCIAS}

1 Justiça do Brasil, Art. 230 do Código de Trânsito Brasileiro - Lei 9503/97. [acesso em 30 julho 2014]. Disponível em:http://www.jusbrasil.com.br/topicos/10600462/artigo-230da-lei-n-9503-de-23-de-setembro-de-1997.

2 Associação Nacional dos Fabricantes de Veículos Automotores - Anfavea. Comércio Exterior. Disponível em: http://www.anfavea.com.br/comercio.html. Acesso em: 14 Abril. 2015.

3 Ambrozin, A. R. P.; Kuri, S. E.; Monteiro, M. R.; Corrosão metálica associada ao uso de combustíveis minerais e biocombustíveis; Revista Quim Nova; 2009; v. 32, no. 7, p. 1910-1916.

4 Guimarães, R.; Filho, J. F. M.; Sensor de corrosão à fibra óptica para poços de petróleo; XV Congresso de Iniciação Científica da UFPE; 2007.

5 Samanez, Carlos Patricio; Ferreira, Léo Da Rocha; Nascimento, Carolina Caldas do. Valuation of the switch input option embedded in the Brazilian flex car: a study of a geographical region using a real options approach and stochastic simulation. Production, v. 24, n. 3, p. 628-643, 2014.

6 Agência Nacional do Petróleo, gás natural e biocombustiveis.; Resolução ANP №40, 2013. [acesso em 11 agosto 2014]. Disponível em:

http://nxt.anp.gov.br/NXT/gateway.dll/leg/resolucoes_anp/2013/outubro/ranp\%2040\%2 0-\%202013.xml. 